Memories of the College of Science, University of Kyoto, Series A, Vol. XXVI, Mathematics No. 2, 1950.

\title{
On the Special Riemann Spaces of Class Two
}

\author{
By
}

\section{Makoto Matsumoto}

(Received Jan. 25, 1950)

In this paper we have presented the special Riemann spaces of class two and type one. In my previous papers ${ }^{(1)}$ I defined the type number of space of class two and gave a necessary and sufficient condition that the space of type $\tau(\geqq 3)$ be of class two. But we have not yet any general research of spaces of type one and two.

Consider a $n$-dimensional variety $V_{n}$ in such an $(n+p)$ - dimensional euclidean space $E_{n+p}$, that the fundamental form

$$
d s^{2}=g_{i j} d x^{i} d x^{j}
$$

is positive definite; and let $B_{p}^{\alpha}(P=I, \ldots \ldots, p ; \mu=1, \ldots, n+p)$ be a system of mutually orthogonal unite vectors normal to $V_{u}$ and put $B_{i}^{\alpha}(i=1, \ldots, n ; \iota=1, \ldots, n+p)=\partial y^{\alpha} / \partial x^{i}$; where $y^{\alpha}$ are the cartesian coordinates in $E_{n+p}$. Then we obtain

$$
\begin{aligned}
& d B_{i}^{\alpha}=\left(\Gamma_{i j}^{k} B_{i}^{\alpha}+H_{i j}^{P} B_{P}^{\alpha}\right) d x^{j}, \\
& d B_{P}^{\alpha}=\left(-g^{k l} H_{l j}^{P} B_{k}^{\alpha}+H_{P j}^{\rho} B_{q}^{\alpha}\right) d x^{j},
\end{aligned}
$$

along a curve on $V_{n}$, where the functions $H_{i j}^{P}$ are symmetric in the indices $i, j$ and called the second fundamentel tensors for the normal $B_{p}^{-\alpha}$; and the functions $H_{q d}^{p}$ are skew-symmetric in the indices $P, Q$. And those functions $H_{i j}^{P}$ and $H_{\ell^{i}}^{P}$ satisfy, moreover, the Gauss eqnation

$$
R_{i j k l}=H_{i k}^{p} H_{j l}^{P}-H_{i l}^{p} H_{j k}^{P},
$$

the Codazzi equation

$$
H_{i j, k}^{P}-H_{i k, j}^{P}=H_{i j}^{q} H_{P k}^{q}-H_{i k}^{q} H_{P j}^{q},
$$

and finally the Ricci equation

$$
H_{q i, j}^{P}-H_{Q j, i}^{P}+\left(H_{Q i}^{R} H_{R j}^{P}-H_{Q j}^{R} H_{R i}^{P}\right)=g^{a b}\left(H_{a i}^{Q} H_{b j}^{P}-H_{a j}^{Q} H_{b i}^{P}\right) .
$$


In particular case for $p=1$ all the functions $H_{q i}^{p}$ vanish identically and in case for $p=2$ the expression in the parenthesis in the left member of (0.4) vanishes identically on account of the skewsymmetric property of $H_{Q i}^{P}$.

When we transform a system of normal vectors $B_{p}^{\alpha}$ to the another $\bar{B}_{P}^{\alpha}$, that is,

$$
\bar{B}_{P}^{\alpha}=l_{p}^{Q} B_{Q}^{\alpha} ; \quad\left|l_{p}^{Q}\right|= \pm 1,
$$

then the second fundamental tensors $H_{i j}^{p}$ are transformed to $\bar{H}_{i j}^{p}$ defined by

$$
\bar{H}_{, i j}^{P}=l_{P}^{Q} H_{i j}^{Q}
$$

Throughout in this paper, by the space we shall mean the real Riemann space whose fundamental form is positive definite.

1. We consider such a $n$-dimensional variety $V_{n}$ of an $(n+2)$ dimensional euclidean space, that, for example, the rank $\tau_{H I}$ of the matrix $\left\|\boldsymbol{H}_{i j}^{\prime \prime}\right\|$ is less than two. Then we have from $(0 \cdot 2)$

$$
R_{i j k l}=H_{i \bar{k}}^{I} \dot{H}_{i l}^{l}-H_{i l}^{l} \dot{H}_{j k}^{I} \text {. }
$$

If $\tau_{11}$ is equal to zero, we get from $(0 \cdot 3)$

$$
H_{i j, k}^{\prime}-H_{i k, j}^{i}=0 \text {, }
$$

so that $V_{n}$ can be imbedded in $(n+1)$-dimensional eucldean space. Consequently if $V_{n}$ is of class two and $\tau_{I l}<2$, we have $\tau_{\mu}=1$. Also, in this case, if the rank $i$ of the matrix $\left\|H_{i j}^{\prime}\right\|$ is more than three, the Codazzi equation $(1 \cdot 2)$ is a result of the Gauss equation $(1 \cdot 1)$ and hence $V_{n}$ is of class one, which was proved by T. Y. Thomas. ${ }^{(9)}$

Now let us find a necessary and sufficient condition that a space $V_{n}$ is of class two, $\tau_{n}$ is equal to one and $\tau_{1}$ is equal to three for a particular choice of a system of normal vectors $B_{p}^{*}$. It has been shown by T. Y. Thomas, ${ }^{(3)}$ that a necessary and sufficient condition for the Gauss equation (1:1) having such a solution $H_{i j}^{\prime}$, that the rank $\tau_{1}$ of the matrix $\left\|H_{i j}^{\prime}\right\|$ is equal to three, be the matrix conditions 
(I) rank of $\left\|R_{1 a b c} \quad R_{2 a b c} \ldots \ldots \ldots R_{n a b c}\right\|=3$,

(II)

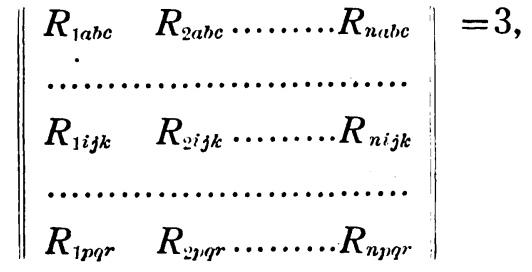

$$
\begin{gathered}
R(a, b, c ; i, j, k) \geqq 0, \\
\sum_{a, b, c, i, i, j, k} R(a, b, c ; i, j, k)>0,
\end{gathered}
$$

and finally

$$
R_{n}(R)=0
$$

where $R(a, b, c ; i, j, k)$ is the determinant

$$
\left|\begin{array}{lll}
R_{a b i j} & R_{a b j j k} & R_{a b k i} \\
R_{b c i j} & R_{b c j k} & R_{b c k i} \\
R_{c c i j} & R_{c a j k} & R_{c a k i}
\end{array}\right|,
$$

and $R_{n}(R)$ is the resultant system of a system of homogeneous equations

$$
\begin{aligned}
& t^{2} R_{i j k l}=H_{i k}^{l} H_{j l}^{l}-H_{i l}^{I} H_{j k}^{l}, \\
& H_{l m}^{l} R_{h i j k}-H_{l k}^{l} R_{h i j m}+H_{j i}^{l} R_{l h m h}-H_{j h}^{l} R_{l k m b}=0,
\end{aligned}
$$

as the above system of equations having a non-trivial solution $\left(t, H_{i j}^{I}\right)$. Then the solution $H_{i j}^{l}$ of the Gauss equation (1.1) is real and uniquely determined to within algebraic sign.

Further, for $V_{n}$ of class two, there must exist two systems of functions $H_{i j}^{I I}\left(=H_{j i}^{I I}\right)$ and $H_{i}(i, j,=1, \ldots \ldots, n)$ satisfying the Codazzi equation

$$
\begin{gathered}
D_{a i j}^{I}=-H_{a i}^{I I} H_{j}+H_{a j}^{I I} H_{i}, \\
D_{a i j}^{I I}=H_{a i}^{I} H_{j}-H_{a j}^{I} H_{i},
\end{gathered}
$$

the Ricci equation

$$
H_{i, j}-H_{j, i}=g^{a b}\left(H_{a i}^{l l} H_{b j}^{l}-H_{a j}^{l 1} H_{b i}^{l}\right),
$$

and finally from $\pi_{l l}=0$

$$
H_{a i}^{\prime \prime} H_{b j}^{\prime \prime}-H_{a j}^{\prime \prime} H_{b i}^{\prime \prime}=0 ;
$$


where $D_{a i j}^{p}$ is defined by

$$
D_{a i j}^{P}=H_{a i, j}^{P}-H_{a j, i \bullet}^{P} .
$$

From $(1 \cdot 3)$ and $(1 \cdot 6)$ we obtain

$$
H_{k r}^{I I} D_{j p q}^{\prime}-H_{j^{r}}^{I I} D_{k p q}^{\prime}=0,
$$

and further easily

$$
D_{a i j}^{\prime} D_{b k l}^{l}-D_{a k l}^{\prime} D_{l i j}^{\prime}=0,
$$

that is a necessary condition.

For the purpose that we obtain the equations determining the functions $H_{i j}^{I I}$, differentiating (1.7) covariantly with respect to $x^{t}$ and summing three equations obtained by permuting the indices $i, j, k$ cyclically, we have

$$
H_{(8} D_{j k) \mid r p q}^{\prime}+H_{r(i}^{I I} D_{j k) \mid p q}^{\prime}=0^{(4)},
$$

on account of (1.4); where $D_{i k \mid r j q q}^{i}$ and $D_{j k \mid \jmath \mu q}^{I}$ are defined by

$$
\begin{aligned}
& D_{j k i r m q}^{\prime}=H_{k r}^{I} D_{j m}^{\prime}-H_{j r}^{l} D_{k \jmath \eta q}^{l}, \\
& D_{j k \mid p q}^{\prime}=D_{j \eta q, k}^{\prime}-D_{k \eta \eta, j}^{z} .
\end{aligned}
$$

Multiplying (1.8) by $H_{h l t}^{\prime \prime}$ and making use of $(1 \cdot 3)$ we obtain

$$
D_{h l(i}^{\prime} D_{j k) \mid r p q}^{\prime}-H_{h l \mid}^{\prime \prime} H_{r(i}^{\prime \prime} D_{j k) \mid p q}^{\prime}=H_{d} H_{h(i}^{\prime \prime} D_{j k) \mid r p q}^{\prime} .
$$

Further multiplying by $D_{a b c}^{\prime}$ and making use of (1.7), (1.9), $(1 \cdot 10)$, (IV) we obtain finally

$$
D_{a b c}^{\prime} D_{h p m}^{\prime} D_{t(i j}^{\prime} H_{k i) r}^{\prime}=H_{h t l}^{\prime \prime} H_{r a}^{\prime \prime} D_{(i|b c|}^{\prime} D_{j k) ! p q}^{\prime} .
$$

In this equation $(1 \cdot 11)$ the quantities $D_{i j k}^{\prime}$ and $H_{i j}^{i}$ are already known.

(A). Assume that all the coefficients $D_{(i|b c|}^{\prime} D_{j k i), p q}^{\prime}$ of $H_{h i c}^{\prime \prime} H_{r a}^{\prime \prime}$ in $(1 \cdot 11)$ vanish. As all $D_{i j k}^{\prime}$ can not be zero, since otherwise $V_{n}$ be of class one, we have

$$
D_{i l(i j}^{u} H_{k i) r}^{\prime}=0 .
$$

We can refer to such a system of coordinates $\left(H^{\prime}\right)$, that at the origin the matrix $\left\|H_{i j}^{\prime}\right\|$ has the form 
On the Special Riemann Spaces of Class Two.

$$
\left\|H_{i j}^{I}\right\|=\left|\begin{array}{ccc}
H_{3}^{I} & \vdots & 0 \\
\ldots \ldots \ldots \ldots \ldots \ldots . . . \\
0 & \vdots & 0
\end{array}\right|, \quad\left|H_{3}^{\prime}\right|=\left|\begin{array}{ccc}
H_{11}^{I} & H_{12}^{I} & H_{13}^{I} \\
H_{21}^{I} & H_{22}^{I} & H_{23}^{I} \\
H_{31}^{I} & H_{i 2}^{I} & H_{33}^{I}
\end{array}\right| \neq 0,
$$

because the matrix $\left\|H_{i j}^{i}\right\|$ is of the rank three. Making the conjugate $H_{i}^{i j}$ of $H_{i j}^{\prime}(i, j=1,2,3)$ in $\left|H_{i}^{\prime}\right|$ and contracting (1.12) for the indices $r, i, j, k=1,2,3 ; d=1, \ldots \ldots, n$ by $H_{1}^{k r}$, we obtain $D_{d i j}^{\prime}=0$ $(i, j=1,2,3 ; d=1, \ldots, n)$. Next taking the indices $r, i, j=1$, 2,$3 ; k>3 ; d=1, \ldots, n$ in $(1.12)$ we have

$$
H_{i r}^{\prime} D_{l i k}^{\prime}+H_{j r}^{\prime} D_{l k i}^{\prime}=0,
$$

and contracting by $H_{i}^{i r}$ we have $D_{l j k}^{t}=0(j=1,2,3 ; k>3 ; d=1, \ldots, n)$. Finally taking the indices $i, r=1,2,3 ; j, k>3 ; d=1, \ldots, n$ in (1.12) we have $D_{t i j k}^{\prime}=0(j, k>3 ; d=1, \ldots n)$. Thus all $D_{a t j}^{\prime}$ are vanishing contrary to hypothesis. Consequently we get, as a necessary condition

$$
\sum_{i, j, k, p, p, q, b, c}^{\prime}\left(\dot{D}_{(i,|b c|}^{\prime} D_{i k)}^{\prime} i_{i m}\right)^{2}>0 .
$$

(B). Next let us prove that a solution $H_{i j}^{\prime \prime}$ of the equation (1·11) is uniquely determined to within algebraic sign. In fact, let $H_{i j}^{i k}$ and $\bar{H}_{i j}^{I I}$ be the two solutions of (1.11) and put

$$
\bar{H}_{i j}^{\prime \prime}=H_{i j}^{\prime \prime}+h_{i j} \text {. }
$$

Substituting (1.13) in (1.11) and making use of (V) we have

$$
h_{h u} h_{r a}+h_{l u d} H_{r u}^{\prime \prime}+H_{l u h}^{\prime \prime} h_{r a}=0 . \quad \text {. }
$$

Assume that the rank of the marix $\left\|h_{i j}\right\|$ is equal to $\tau(\neq 0)$, and refer to a system of coordinates that at the origin the matrix $\left\|h_{i j}\right\|$ has the form

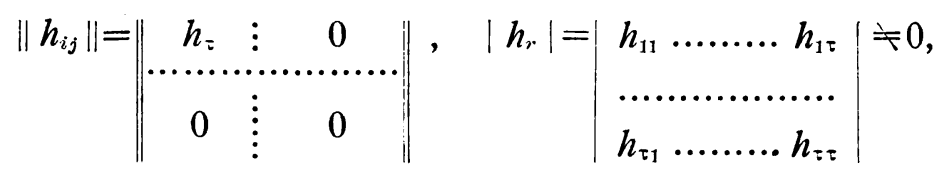

and make the conjugate $h^{i j}$ of $h_{i j}(i, j=1, \ldots, \tau)$ in $\left|h_{\tau}\right|$. Contracing (1.14) for the indices $h, d, r, a=1, \ldots, \tau$ by $h^{h l l}$ gives

$$
\left(\tau+h^{h l d} H_{h l d}^{\prime I}\right) h_{r a}+\tau H_{r a}^{\prime r}=0 .
$$


Further contracting by $h^{\text {ra }}$ gives $h^{h l} H_{h l}^{\prime \prime}=-\tau / 2$, so that from (1.15) we have $h_{r a}=-2 H_{r a}^{\prime \prime}$, and then from $(1 \cdot 13) \tilde{H}_{i j}^{\prime \prime}=-H_{i j}^{\prime \prime}$ $(i, j=1, \ldots, \tau)$, Next taking the indices $h, d, a=1, \ldots, \tau ; r>\tau$ in (1.14) we have $H_{r a}^{\prime \prime}=0(r>\tau ; a=1 \ldots, \tau)$ and taking the indices $h, d=1, \ldots, \tau ; r, a>\tau$ we have $H_{r a}^{\prime \prime}=0(r, a>\tau)$, so that $\dot{H}_{r a}^{\prime \prime}=0$ $(a=1, \ldots, n ; r>\tau)$. Hence we see $\bar{H}_{i j}^{\prime \prime}=-H_{i j}^{\prime \prime}(i, j=1, \ldots n)$ for $i \neq 0$ and the above statement is proved.

(C.) Next, let us find a necessary and sufficient condition that the solution $H_{i j}^{\prime \prime}$ is real. Taking the indices $r$ and $a$ equal to $h$ and $d$ respectively in $(1 \cdot 11)$ gives

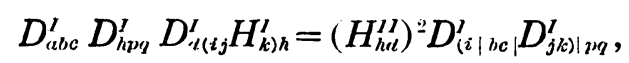

and hence we have

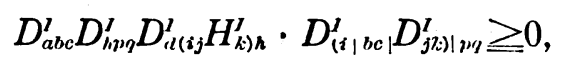

that is a necessary condition. Conversely we see easily that if (VI) is satisfied, neither of these salution $H_{i j}^{\prime \prime}$ can be pure imaginary.

Now we put

$$
H_{i i}^{\prime \prime}=p_{i j}+\sqrt{-1} \quad q_{i j} ;
$$

where the $p$ 's and $q$ 's are all real. 'Substituting from (1.16) in $(1 \cdot 11)$ and equating the imaginary part to zero we obtain

$$
p_{h t} q_{r a}+q_{h t} p_{r a}=0 \text {. }
$$

By the similar process which was used in (B), it follows from $(1 \cdot 17)$ that if the rank of the matrix $\left\|q_{i j}\right\|$ does not vanish, all the $p_{i j}$ are equal to zero, so that the solution $H_{i j}^{\prime \prime}$ is real or pure imaginary. Consequently if (VI) is satisfied, the solution is real.

(D.) Finally we see easily that the necessary and sufficient condition for the system of equation $(1 \cdot 11)$ to have a solution $H_{i j}^{\prime \prime}$, is (VII)

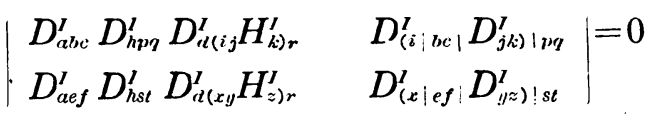

that is the resultant system of the system of homogeneous equations

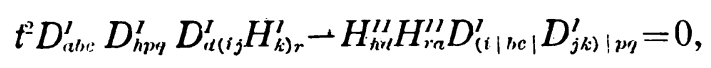


having a non-trivial solution $\left(t, H_{i j}^{\prime \prime}\right)$.

Conversely if the conditions (V), (VI); (VII) are satisfied, it is easily seen that $(1 \cdot 18)$ has a non-trivial solution $\left(t, H_{i j}^{l k}\right)$; where $t \div 0$, and thus $H_{i j}^{\prime \prime} / t$ is a solution of $(1 \cdot 11)$ and also is real. Hence the functions $H_{i, j}^{\prime \prime}$ are uniquely determined to within algebraic sign; for example, when

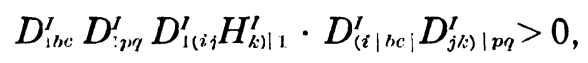

we have

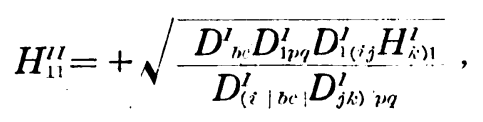

and for another $H_{l i d}^{\prime \prime}$ we have

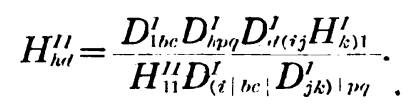

(E). From (1-11) we have by means of (IV)

$$
H_{u l}^{\prime \prime} H_{r a}^{\prime \prime}-H_{l u t}^{\prime \prime} H_{r l}^{\prime \prime}=0 \text {, }
$$

so that the condition $(1 \cdot 6)$ is satisfied.

II. Now, for the purpose that we get the functions $H_{i}(i=1, \ldots$, $n$ ) satisfying the equation $(1 \cdot 4)$, consider a system of homogeneous equations

$$
t D_{a i j}^{\prime \prime}-H_{a i}^{\prime} H_{j}+H_{a j}^{\prime} H_{i}=0,
$$

where $t, H_{i}(i=1, \ldots, n)$ are unknown. We see easily that all of $H_{i}$ can not be zero for $V_{n}$ of class two. Therefore the equation $(2 \cdot 1)$ must have a non-trivial solution $\left(t, H_{i}\right)$, so that we have a matrix condition;

rank of

(VIII)

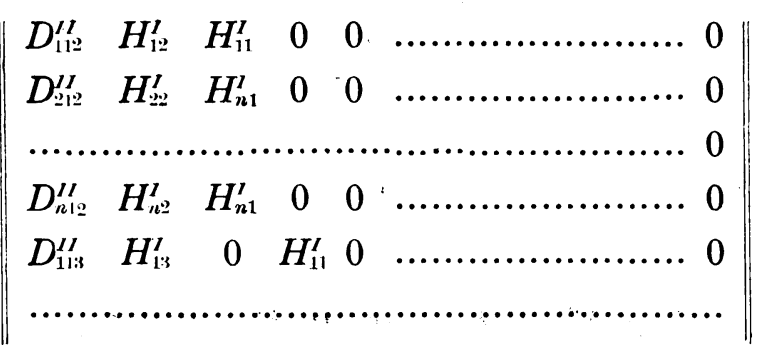




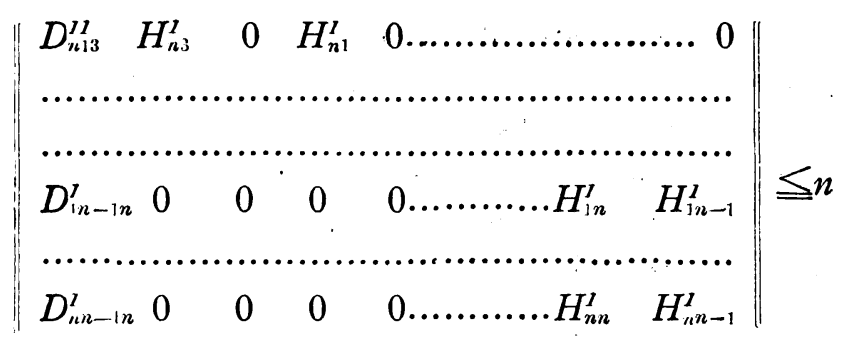

as a necessary condition. Conversely, if the condition (VIII) is satisfied, the system of equations $(2 \cdot 1)$ has a non-trivial solution $\left(t, H_{i}\right)$, where $t$ is not vanishing; that is proved easily, putting $t=0$ in $(2 \cdot 1) i, e$.

$$
H_{a i}^{\prime} H_{j}-H_{a j}^{\prime} H_{i}=0 \text {, }
$$

and refering to the system of coordinates $\left(H^{\prime}\right)$. Therefore we have $H_{i} / t$ satisfying the system of equations $(1 \cdot 4)$. Also, in the similar manner, it is to be seen easily that the solutian $H_{i}$ is uniquely determined and is real. Explicitly we have the form $H_{i}$, refering to the system of coordinates $\left(H^{I}\right)$, as follows,

$$
\begin{aligned}
& H_{i}=\frac{1}{2} H_{\lrcorner}^{a l} D_{a k i}^{\prime l} \quad(a, b, i=1,2,3), \\
& H_{i} H_{j k}^{\prime}=D_{j k i}^{\prime \prime} \quad(i>3 ; j, k=1,2,3),
\end{aligned}
$$

where the indices $j, k$ of $H_{j k}^{\prime}$ are to be chosen for $H_{j k}^{\prime} \neq 0$.

Thus obtained $H_{i j}^{i}, H_{i j}^{l I}$ and $H_{i}(i, j=1,=, n)$ must satisfy the equation $(1 \cdot 3), i . e$.

$$
D_{a i j}^{\prime}+H_{a i}^{11} H_{j}-H_{a j}^{\prime \prime} H_{i}=0,
$$

that is a necessary condition.

Now we remark that those $H_{i j}^{\prime}, H_{i j}^{\prime l}$ and $H_{i}$ satisfy the Ricci equation (1.5). In fact, differentiating (1.4) covariantly with respect to $x^{k}$ and summing three equations obtained by cyclic permutation of the indices $i, j, k$ give by means of (IX) and $(1 \cdot 2)$

$$
H_{a(s}^{\prime} D_{j k)}=0,
$$

where

$$
D_{j k}=H_{j, k}-H_{k, j}-g^{a b}\left(H_{a j}^{\prime \prime} H_{b k}^{\prime}-H_{a k}^{\prime \prime} H_{b j}^{\prime}\right) .
$$


On he tSpecial Riemann Spaces of Class Two.

We have immediately $D_{j k}=0$, refering to the system of coordinates $\left(H^{\prime}\right)$.

Consequently we obtain the

Theorem : ......A Riemann space $V_{n}$ of dimensionality $n(\geqq 3)$ is of class two and the matrices $\left\|H_{i j}^{i}\right\|$ and $\left\|H_{i j}^{\prime \prime}\right\|$, whose elements are the second fundamental tensors of $V_{n}$, are equal to three and one respectively for a particular choice of a system of normal vectors if, and only if, the inequalities (II), (V), (VI) and the equations (III), (IV), (VII), (IX) and finally the matrix conditions (I), (VIII) are satisfied.

Finally it is to be noted that the curvature tensor $R_{i j k l}$ of those $V_{n}$ satisfies the equation

$$
R_{a . i(j)}^{b} R_{|b| \cdot k l)}^{a}=0,
$$

making use on $(1 \cdot 1)$ and therefore $V_{n}$ is of type one. ${ }^{(5)}$ But those $V_{n}$ are not the general spaces of class two ane type one.

\section{References}

(1) Jour. Japan Math. So., vol. 2. nos. 1-2. Riemann spaces of class two and their algebraic characterization. pp. 67-92.

(2) Acta Mate., 61 (1936) p. 189

(3) 1. c., p. 194

(4) for example

$$
A_{(i|b|} B_{\left.j_{k j}\right)}=A_{i b} B_{j_{k}}+A_{j_{b}} B_{k i}+A_{k b} B_{i j}
$$

(5) M. Matsumoto, 1. c., p. 69 